\title{
Effect of information about COVID-19 vaccine effectiveness and side effects on behavioural intentions: two online experiments
}

3 Department of Public Health and Primary Care, University of Cambridge

* Correspondence: jk803@cam.ac.uk

Citation: Lastname, F.; Lastname, F.; Lastname, F. Title. Vaccines 2021, 9 , x. https://doi.org/10.3390/xxxxx

Academic Editor: Firstname Lastname

\section{Received: date}

\section{Accepted: date}

Published: date

Publisher's Note: MDPI stays neutral with regard to jurisdictional claims in published maps and institutional affiliations.

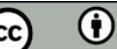

Copyright: () 2021 by the authors. Submitted for possible open access publication under the terms and conditions of the Creative Commons Attribution (CC BY) license (http://creativecommons.org/licenses (by/4.0/).

\begin{abstract}
The success of mass COVID-19 vaccination campaigns rests on widespread uptake. However, although vaccinations provide good protection, they do not offer full immunity and while they likely reduce transmission of the virus to others, the extent of this remains uncertain. This produces a dilemma for communicators who wish to be transparent about benefits and harms and encourage continued caution in vaccinated individuals but not undermine confidence in an important public health measure. In two large pre-registered experimental studies on quota-sampled UK public participants we investigate the effects of providing transparent communication-including uncertainty-about vaccination effectiveness on decision-making. In Study 1 ( $n=2,097)$ we report that detailed information about COVID-19 vaccines, including results of clinical trials, does not have a significant impact on beliefs about the efficacy of such vaccines, concerns over side effects, or intentions to receive a vaccine. Study $2(n=2,217)$ addressed concerns that highlighting the need to maintain protective behaviours (e.g. social distancing) post-vaccination may lower perceptions of vaccine efficacy and willingness to receive a vaccine. We do not find evidence of this: transparent messages did not significantly reduce perceptions of vaccine efficacy, and in some cases increased perceptions of efficacy. We again report no main effect of messages on intentions to receive a vaccine. The results of both studies suggest that transparently informing people of the limitations of vaccinations does not reduce intentions to be vaccinated but neither does it increase intentions to engage in protective behaviours post-vaccination.
\end{abstract}

Keywords: COVID-19, vaccination, hesitancy, communication, vaccine messaging

\section{How to Use This Template}

\section{Introduction}

At the time of writing more than 2.5 million people are estimated to have died from COVID-19 [1] and wide-ranging restrictions have been placed on people across the globe, with immense social and economic consequences [2,3]. Mass vaccination has been heralded as a 'checkmate' move to end the pandemic [4]. However for lasting and widespread protection, this relies firstly on vaccines being developed that significantly reduce transmission of the disease rather than simple individual protection, and secondly, sufficient uptake of the vaccine to produce 'herd immunity' - a level of population immunity at which an infectious agent is no longer able to spread [5]. Estimates of the percentage of the population required to be vaccinated to achieve this goal vary (and depend on the local reproduction rate, $R$, of the virus), with most estimates in the $60-80 \%$ range [6,7].

A sizable minority of people around the world express hesitancy towards taking COVID-19 vaccines [8]-defined as "delay in acceptance or refusal of vaccines despite 
availability of vaccination services." [9]. Factors such as religion, gender, political ideology, and trust in medical and scientific institutions have been shown to be associated with vaccine hesitancy, both in general and regarding COVID-19 vaccines specifically $[8,10,11]$. While these broader factors are important, research has also shown that specific beliefs about and attitudes towards COVID-19 vaccinations are also closely linked to vaccination intentions $[8,12]$. Whether these attitudes can be, and should be, changed by communication 'campaigns' is a matter of active debate worldwide.

\subsection{Transparent communication of vaccine risks and benefits}

Many public health commentators and researchers have called for transparent communication of COVID-19 vaccine information, including the efficacy and side effects reported from clinical trials, to improve vaccine uptake [13-15]. Some, for example, Rzymski et al. [16], think that such information may change individuals' vaccine beliefs: "The general public must be given access to the pivotal information on the authorized vaccines, and that their approval is based on the evidenced benefits that outweigh the potential risks of vaccine administration" (p. 3). Others see such transparency as more a matter of ensuring public trust in science, or simply an ethical imperative [17]. Such calls demand empirical evidence of their potential effects, and hence are the impetus for the current study. What are the effects of such communications on individuals' beliefs about COVID19 and their intentions to receive a vaccine? We note three important considerations in approaching this question.

Firstly, vaccination is an invasive medical procedure [18] with well-defined (if small) risks to the individual. As such, in some countries it is treated as an individual medical decision and requires informed consent (in others it is treated as a public health policy and can be mandated without the option of consent or refusal - for some discussion see [19-22]). In the case of the COVID-19 vaccines, until recently there was no evidence that they reduced transmission of the disease to others and therefore were more accurately considered a method of individual rather than public protection. Many might argue, therefore, that clearly and transparently communicating what is currently known about COVID-19 vaccines should be considered important from an ethical [23] (and potential legal [24]) standpoint, regardless of any expected effect of such messages on individuals' vaccination intentions.

Second, it is currently unclear that transparent communication of COVID-19 vaccine information would necessarily affect vaccination rates in any predictable way. On the one hand, explaining the results of clinical trials and the regulatory procedures in place may serve to alleviate concerns over vaccine efficacy and safety and ultimately increase intention to vaccinate as suggested by some researchers [12]. On the other, as noted by Petersen et al. [25], it is also possible that individuals may find the information more concerning, increasing their perception of vaccines as relatively more dangerous or ineffective. That is, some people may find the reported frequency of COVID-19 vaccine side effects to be higher, or efficacy of vaccines to be lower than they expected. Indeed, some (pre-COVID) vaccination messaging studies have reported 'backfire' effects, such that well-meaning efforts to increase vaccination intentions actually increase safety concerns and lower vaccination intentions [26].

Third, the notion that COVID-19 vaccine hesitancy is the product of a lack of information reflects a 'deficit model' approach to addressing the overarching issue. That is, it assumes simply providing information will lead to attitudes and behaviours in line with the mainstream scientific position [27]. In the context of vaccination attitudes, Kitta and Goldberg [28] position the deficit model as "...the presumption that vaccination opposition and/or refusals are primarily driven by insufficient understanding of the facts regarding vaccination risks and benefits." (p. 2). Within science and health communication fields the deficit model approach has received widespread criticism as ignoring the myriad social, cultural and psychological factors that drive risk perceptions and attitudes toward medical and scientific issues and technologies [28]. However, COVID-19 vaccines are a 
very new medical intervention and a lack of information may play a greater role in attitudes and intentions compared to resistance towards other, more established vaccines. This point is explicitly noted by Opel et al. [29] in comparing general vaccine hesitancy to COVID-19 vaccine hesitancy. They write: "The former is steeped in a complex sociocultural milieu of consumerism, pervasive misinformation, and the rejection of objective, scientific evidence as truth. The latter may simply represent a deficit of the data needed to make an informed decision." (p. 1)

Regardless of the considerations outlined above, public health authorities around the world are currently communicating information about COVID-19 vaccines to citizens. Thus we conducted these experiments with the intention of generating empirical data on the effects of such real-world communications, in order to inform debates about the best kinds of communication to serve the intentions of different communicators, whether they be attempting to inform or persuade.

\subsection{Beliefs about COVID vaccines and vaccine hesitancy.}

Numerous cross-sectional surveys around the world have identified perceptions of COVID-19 vaccines as safe and effective as key predictors of COVID-19 vaccination intentions (e.g., [12,30-35]). Indeed, a recent systematic review of such studies [8] concludes that perceptions of vaccine safety and effectiveness are 'universally' consistent determinants of COVID-19 vaccine hesitancy (p. 8). Further, surveys which specifically asked COVID-19 vaccine hesitant participants their reason for vaccine refusal or delay consistently report that concerns over safety and efficacy are among the most common reasons given [33,36-40]. Similarly, qualitative research has also highlighted these concerns as reported drivers of COVID-19 vaccine hesitancy among minority and at-risk groups [4143]. Such concerns may be fuelled by COVID-19 vaccine misinformation [44] and recent research has identified susceptibility to misinformation as a correlate of COVID-19 vaccine hesitancy [45].

Such findings align with Health Belief Model theoretical framework which positions the perceived risks and benefits of a given health behaviour as key predictors of intentions to engage in that behaviour (alongside perceptions of disease threat) [46,47]. The Health Belief Model has informed previous vaccine communication research. For example, Jones et al. [48] show that exposure to vaccination campaign advertisements is associated with increased willingness to receive a H1N1 vaccine, and that this effect is partially mediated by belief that the vaccine will prevent disease and belief that the vaccine would not have a negative impact on one's health. The authors conclude that addressing perceived barriers (e.g. perceptions of vaccine risk) may be the most effective target for interventions to improve vaccine uptake.

\subsection{The effect of COVID-19 vaccine communications on intentions}

Few studies have experimentally investigated the effects of COVID-19 vaccine information on related attitudes and intentions, and most have focused on hypothetical vaccines due to the lack of approved vaccines at the time of research. In discrete choice-type experiments, participants unsurprisingly express greater preference for more effective (hypothetical) vaccines with fewer side effects, ceteris paribus [49-53]. However, experimental studies which have examined the impact of COVID-19 vaccine information on behavioural intentions report mixed results. For example Petersen et al. [25] report that transparent positive messages heralding a safe and effective fictional COVID-19 vaccine (70\% efficacy with a 1 in 10,000 incidence of serious side effects) decreased vaccine scepticism relative to vague or negative transparent messages. However, no control group was included in the study-leaving it unclear which interventions shifted beliefs relative to a baseline. In another messaging experiment, Motta et al. [54] report that messages framed to emphasise the collective benefits of vaccination or the risks of not vaccinating increased participants' vaccine intentions - however the presence or absence of information about the rigorousness of clinical trials had no impact on intentions. Palm et al. [55] find that messages highlighting vaccine efficacy and safety had a marginally significant effect on 
vaccine intentions relative to a control group, while an opposite message claiming planned vaccines were neither effective nor safe had no main effect on intentions. Conversely, in a pre-post design experiment Loomba et al. [56] report that exposure to a range of factual information about potential COVID-19 vaccines has no effect on vaccine intentions, while COVID-19 vaccine misinformation decreases vaccination intentions. Duquette [57] reports that short messages framing vaccination as protecting either oneself or others have no effect on reported vaccination intentions (but notes that there are some experimental effects among non-white participants).

Notably, all of the studies reviewed above focus on fictional or hypothetical vaccines, and none examine the impact of information on beliefs about COVID-19 vaccines - such as perceptions of efficacy or safety - separately from intentions to receive a vaccine. We are aware of only one study which has used actual results from clinical trials as part of an experimental intervention. Altay et al. [58] paid French participants to spend 10 minutes interacting with an extensive Q\&A interface in which users could select questions and read answers (the complete Q\&A comprised more than 20 pages of text). Some of the answers included preliminary results from clinical trials outlining vaccine efficacy ( $95 \%$ for Pfizer/BioNTech and Moderna, and $<70 \%$ for AstraZeneca) and noted some side effects without giving any indication of frequency. Altay et al. report that participants who interacted with the Q\&A were more likely to report intentions to receive a COVID-19 vaccine when available and expressed more positive attitudes towards vaccines, compared to a control group. However, it is unknown how many participants actually accessed or read the information on vaccine safety and efficacy.

In Study 1, we examine the effects of reading comprehensive information from recent clinical trials (in two different formats) on individuals' intentions to receive a vaccine. In addition to investigating vaccination intentions we also include several outcome measures examining: participants' belief that COVID-19 vaccines are effective, concern over side effects and concern over speed of regulatory approval (using the Oxford COVID-19 Vaccine Beliefs Scale [12]). We also include two further exploratory messages as experimental conditions. The first focuses on addressing concerns over the speed of vaccine development and approval, which is also associated with vaccine hesitancy [12] and the second presents a mechanistic description of how vaccines, including the new mRNA vaccines, work. Mechanistic explanations have been shown to reduce risk perceptions and shift attitudes in other scientific domains [59,60].

\subsection{Post-vaccination protective behaviour messaging and vaccine confidence}

Although the reported efficacy of some vaccines in preventing SARS-CoV-2 symptoms is very high (e.g. 94.1\% for the Moderna mRNA vaccine [61]), there is considerable uncertainty about the effectiveness of others, particularly in some groups of people (e.g. AstraZeneca in over-65s [62]). At the time of writing, the extent to which vaccines will prevent transmission of the virus is even more unclear and the majority of people in most countries are unvaccinated [63]. This has lead public health authorities to emphasise the need for vaccinated individuals to continue engaging to preventative behaviours such as social distancing, frequent handwashing and wearing a mask in public spaces to prevent the spread of the virus [64-66].

Do such messages increase intention to continue protective behaviours post-vaccination? And do they have unintended consequences? It is possible that such messaging could lead individuals to think that vaccines are ineffective, and/or reduce intentions to receive a vaccine as some perceived benefits (e.g. not having to wear a mask or social distance) are no longer present. As Savulescu [67] speculates, freedom from social distancing and mask wearing requirements may be a powerful personal incentive for some individuals to receive a COVID-19 vaccine (see also [68]). Equally, could health messages in use to encourage the uptake of the vaccine which minimise uncertainty and risks (e.g. 'vaccines are safe and effective') encourage vaccinated people to stop engaging in protective behaviours once vaccinated? 
In Study 2 we test these possibilities, providing participants with messages encouraging vaccination, with or without caveats about effectiveness and additional reminders of the need to maintain protective behaviours once vaccinated.

\section{Study 1}

This study sought to answer the following research question: does information specifically targeted to beliefs about vaccine efficacy, or concerns about safety shift these beliefs, and, if so, do these changes in turn have an impact on COVID-19 vaccination intentions? To this end, we conducted a survey experiment[69], in we which we randomly allocate participants to read different messages or no message at all, before measuring attitude and behavioural intentions. This methodology allows us to clearly establish cause and effect when examining the impact of different messages, and follows from a wellestablished approach used many previous vaccine messaging studies (e.g., [54,70,71]) . By conducting the survey online (vs. in a laboratory setting), we were able to recruit a large and broadly representative sample. We note that the different messages presented here were drawn from publicly available information and do not vary systematically in content, thus our main focus is comparing the different messages with the control group. We would also add that none of the messages were directly persuasive regarding vaccination, that is, none explicitly stated that the reader should accept a vaccine if offered. This study was pre-registered (https://aspredicted.org/blind.php?x=we7am9) and ethical oversight was provided by the Psychology Research Ethics Committee at the University of Cambridge (PRE.2020.034 with amendment on $9^{\text {th }}$ January 2021).

\subsection{Methods}

\subsubsection{Participants}

An a priori power calculation performed using GPOWER [72] indicated a total sample size of at least $\mathrm{N}=1,865$ would be required to detect an effect size of $\mathrm{f}=0.1$ in a oneway ANOVA with five groups, at $95 \%$ power and an alpha level of .05. We deliberately recruited a larger sample to account for the exclusion criteria detailed below.

Adult participants resident in the U.K. were recruited through two opt-in online panels[73]: Respondi (Respondi.com) and Prolific (prolific.co), between 13-17 January 2021. These web-based platforms facilitate recruitment of participants by emailing or messaging individuals registered with the platform about available studies in which they participate. Participants receive compensation for completing studies (usually in the range of \$2-3 US). Both panels are large, with each reporting more than 50,000 potential UK participants. Participants recruited via Respondi were quota sampled (using Qualtrics survey software) to match the age and sex distribution of the UK population. Participants recruited via Prolific were quota sampled by the platform to match the UK population in terms of age, sex, and ethnicity [74].

A total of 2,488 participants completed the survey (Respondi $n=1,233$; Prolific $n=$ 1,255). As per our pre-registration, 391 participants were excluded for the following reasons: failing an instructional attention check ('Please select 'somewhat agree' ...', n = 279), reporting having already received a COVID-19 vaccine $(n=90)$, providing an age under $18(n=2)$ or higher than $100(n=3)$.

This resulted in a final sample for analysis of 2,097 $\left(M_{\text {Age }}=43.2, S D=15.5 ; 51.7 \%\right.$ female; median highest education: Bachelors degree or equivalent; $87 \%$ White ethnicity).

\subsubsection{Materials and procedure}

After providing consent, participants answered questions about their attitudes and beliefs around COVID-19 before being randomised via the Qualtrics randomisation function to an experimental group and answer questions about their COVID-19 vaccine beliefs and intentions. They were blinded to the randomisation. All participants then completed a section of demographic questions. 
Information Texts. There were four different information conditions and one control condition in the experiment. Participants in the control condition were presented with no information, but instead proceeded directly to post-information items. Participants in all other conditions were presented with information on a single webpage within the survey. All started with the following text: "Several new vaccines have been developed to protect people against COVID-19, which is the disease caused by the SARS-Cov2 coronavirus. These vaccines have been developed and tested throughout 2020 and are now becoming available to the public."

The content of each text is briefly summarised in Table 1 ; the full text of each condition can be found in the supplementary information (Appendix 1). The Factbox format is a tabular format allowing people to compare potential benefits and harms in those taking and not taking a particular medical treatment. It has been shown to help people understand and remember such information [75-77]. The text $\mathbf{Q} \& \mathbf{A}$ format was an edited version of text from the European Medicines Agency website. Both were designed to address concerns about efficacy and side effects, but the Factbox condition was much more explicit about side effects. Data reported in the Factbox and Q\&A conditions was based on a trial of the Moderna vaccine, but the name was omitted from the text. No source for the information was given. The regulatory Approval message condition gave participants information taken from the European Medicines Agency website specifically designed to address concerns about the accelerated approval process of the COVID-19 vaccines. The scientific Mechanism message condition gave participants information about how vaccines, and in particular the mRNA vaccines developed against COVID-19, work, designed to address misinformation and misunderstandings about the mechanism and contents of the vaccines, given previous findings on the effect of a meaningful mechanistic explanation (Schneider et al., in preperation).

Table 1: Overview of message content in experimental conditions

\begin{tabular}{lcc}
\hline $\begin{array}{c}\text { Condi- } \\
\text { tion }\end{array}$ & $\mathbf{n}$ & Content \\
\hline Control & 412 & None \\
Factbox & 409 & $\begin{array}{c}\text { Tables detailing incidence of COVID-19 } \\
\text { and side effects in the vaccine and pla- } \\
\text { cebo arms of a large clinical trial, both as } \\
\text { absolute numbers and percentages. Data } \\
\text { was presented separately for trial partici- } \\
\text { pants aged 18-65 and aged 65+. A sepa- } \\
\text { rate table reported rare side effects. }\end{array}$ \\
\hline
\end{tabular}

Q\&A $417 \quad$ A Q\&A format outlining the results of a clinical trial, including the absolute numbers of symptomatic COVID- 19 cases and vaccine efficacy. The text also noted several recommendations around the vaccine and known side effects with approximate frequencies, ranging from mild (e.g. pain at injection site, 1 in 10) to severe (facial swelling and facial paraly-

Adapted from US

541 FDA [79] briefing document

Length in words

(incl. ta-

bles)

-

European Medi708 cines Agency [80] webpage 


\begin{tabular}{|c|c|c|c|c|}
\hline $\begin{array}{c}\text { Ap- } \\
\text { proval }\end{array}$ & 443 & $\begin{array}{l}\text { An overview of the standard and expe- } \\
\text { dited COVID-19 vaccine review pro- } \\
\text { cesses, highlighting the point that regula- } \\
\text { tory assessments of COVID-19 vaccine } \\
\text { data were undertaken during the re- } \\
\text { search and development process, as op- } \\
\text { posed after. A figure depicted this visu- } \\
\text { ally. }\end{array}$ & $\begin{array}{l}\text { European Medi- } \\
\text { cines Agency [81] } \\
\text { webpage }\end{array}$ & 624 \\
\hline $\begin{array}{c}\text { Mecha- } \\
\text { nism }\end{array}$ & 416 & $\begin{array}{l}\text { A description of how vaccines induce } \\
\text { immunity and in particular the mecha- } \\
\text { nism by which mRNA vaccines produce } \\
\text { antigens, noting the benefits of using } \\
\text { mRNA, such scaling up vaccine produc- } \\
\text { tion quickly. Two figures were included. }\end{array}$ & $\begin{array}{l}\text { Extracts from US } \\
\text { CDC [82] and } \\
\text { British Society for } \\
\text { Immunology [83] } \\
\text { and a figure from } \\
\text { Pfizer }\end{array}$ & 686 \\
\hline
\end{tabular}

As the information presented in the conditions was taken from different sources, the content and format varied widely. In particular, we note that, although based on the same trial, efficacy figures in the Factbox and Q\&A conditions differed slightly. In the Factbox condition, efficacy was reported separately for trial participants aged 18-65 and 65 and older (95.5\% and $86.2 \%$ respectively) while the Q\&A reported an overall efficacy of $94.1 \%$. The Q\&A text also only gave a broad account of side effects, e.g. that mild and moderate side effects "affected more than 1 in 10 people". This can be compared with the specific side effect frequencies reported in the Factbox text, including the relatively high rate of reported pain at the injection site among vaccinated individuals (e.g. $18.8 \%$ in the placebo group and $90.1 \%$ in the vaccine group, among 18-64 year olds).

Primary Outcomes. Participants completed the seven item Oxford COVID-19 Vaccine Hesitancy Scale, which aims to "assess expressed intent to accept a COVID-19 vaccine" (Freeman et al., 2021, p. 2; example item: I would describe my attitude towards receiving a COVID-19 vaccine as; response scale: 1 = Pretty keen, $5=$ Against it; $\quad \alpha=.97)$. The scale was reversed such that higher values indicate a greater willingness or intention to receive a vaccine.

Participants also completed an adapted version of the Oxford COVID-19 Vaccine Beliefs Scale [12] including the subscales capturing: belief that COVID-19 vaccines are effective (The COVID-19 vaccine is likely to; $1=$ Definitely work for me , 5 = Definitely not work for $m e ; \alpha=.87$ ), concern over COVID-19 side-effects (The side effects for people of getting the COVID-19 vaccine will be; $1=$ None, $5=$ Life threatening; $\alpha=.76$ ), and concern about the speed of vaccine development and approval (The speed of developing and testing the vaccine means it will be; $1=$ Really safe, $5=$ Really unsafe; $\alpha=.79)$. Scores on the efficacy scale were reversed such that higher values indicate a perception that COVID-19 vaccines are effective. The original Freeman et al. [12] scale dimensions were based on exploratory and confirmatory factor analyses of a larger pool of items. Results from these original analyses indicated some scale items which only loaded weakly onto the identified factors (e.g. a risk question about likelihood of infection had a loading of .45 on the efficacy factor). In light of this, we added one or two face-valid items to each scale and several items were removed (see Appendix 2 for a full description of changes). As expected, the adapted subscales displayed higher reliability than the original scales (however, analyses using the original scale items produced essentially identical results; see Appendix 2).

Secondary Outcomes. Participants also completed a second, binary measure of vaccination intention, responding Yes or No to the question: 'If you were offered a COVID-19 vaccine would you get vaccinated yourself?' All participants completed two measures adapted from the Decisional Conflict Scale [84], with all items prefaced with the stem: 'If 
you were given the option of receiving a COVID-19 vaccine or not today how would you feel about the decision?'. The first was a three-item measure of feeling informed regarding the decision to vaccinate (example: I would feel I had made an informed choice; $1=$ Strongly disagree, $5=$ Strongly agree; $\alpha=.79)$. The second was a three-item measure of decision certainty ( $I$ feel sure about what to choose; $1=$ Strongly disagree, $5=$ Strongly agree; $\alpha=.92)^{1}$.

All participants also were asked to estimate the frequency of mild to moderate and severe side effects of COVID-19 vaccines (per 10,000 people vaccinated; this denominator was chosen to align with the figures reported in the Factbox and Q\&A conditions). Participants then provided an estimate of efficacy of the 'most effective COVID-19 vaccine available' (By how much do you think the most effective COVID-19 vaccine available reduces the chance of someone becoming ill with the disease?). Participants entered their estimate on a sliding scale with anchors ' $0 \%$ - Doesn't make any difference' and '100\% - Stops all COVID-19 disease'. We refer to this variable as 'estimated efficacy' to distinguish it from the perceived efficacy primary outcome,

Participants in the information conditions (i.e. all except control) also completed several measures assessing their perceptions of the information they had just read. These were measures of: how trustworthy (three items, $\alpha=.96$ ), engaging (three items, $\alpha=.83$ ), and believable they found the information; how much they felt they understood the information; the level of effort required to read the information; and the perceived quality of the evidence underlying the information.

\subsection{Results}

Participants, on average, reported the messages to be relatively trustworthy (Ms 5.205.61, SDs 1.16-1.33), engaging (Ms 5.17-5.68, SDs 1.28-1.36) and believable (Ms 5.32-5.63, $S D$ s 1.21-1.37), with mean scores on these measures above the scale mid-point (range 1-7 ) across all message conditions (see Appendix 3 for comparisons between conditions).

\subsubsection{Primary outcomes}

Reading detailed information about the risks and benefits of vaccination, the vaccine approval process, or how vaccines induce immunity had no significant impact on the main dependent variables: one-way ANOVAs revealed no significant effect of experimental condition on willingness to receive a COVID-19 vaccine, $F(4,2091)=0.72, p=.58$; belief that COVID-19 vaccines are effective, $F(4,2092)=0.63, p=.64$, concern over COVID19 vaccine side effects, $F(4,2091)=1.51, p=.20$, or concern over the speed of COVID vaccine regulatory approval, $F(4,2090)=0.71, p=.58$. Results are shown graphically in Figure 1 , and descriptive statistics for all outcomes are reported in Appendix 3.

1 The six Decisional Conflict Scale (DCS) items were selected as the most relevant to the COVID-19 vaccine decision and we had intended treat these as a single scale $(\alpha=.90)$. However parallel and exploratory factor analyses revealed two factors differentially impacted by the experimental intervention. This lead to the creation of the two ad hoc two scales reported here (a deviation from our pre-registration). With regards to the original DCS factor structure, the three certainty items come from the Uncertainty subscale, whereas the informed items in the current study comprise two items from the Informed subscale (knowing the risks and benefits) and one from the Effective Decision subscale ('I would feel I had made an informed choice'). 

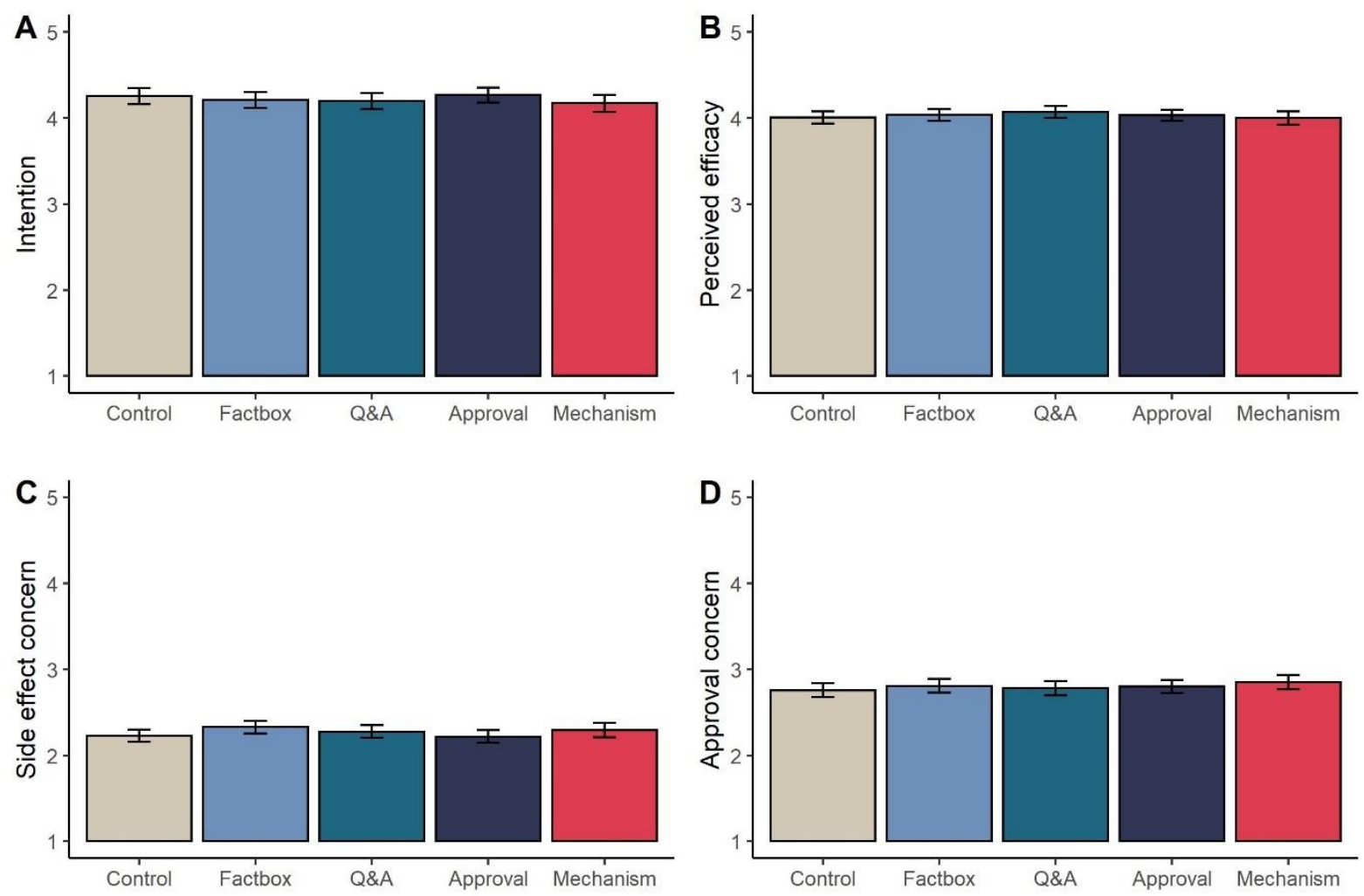

\section{Condition}

Figure 1: Mean levels (95\%CI) of (A) intention to receive a COVID-19 vaccine, (B) perceived efficacy of COVID-19 vaccines, (C) concern over COVID-19 side effects, and (D) concern over speed of COVID-19 vaccine approval process, across the five experimental conditions.

Given the absence of statistically significant effects, we also conducted non-preregistered exploratory Bayesian ANOVAs to investigate the extent to which the data supports a null model (no effect of condition) over a main effect model in which condition does have an effect on the outcome variables. Bayes factors from comparison of models analysing vaccine intentions $(B F=1013.40)$, perceived efficacy $(B F=1199.42)$, concern over side effects $(B F=223.48)$, and concern over speed of approval $(B F=1026.08)$ all indicated 'very strong' or 'decisive' evidence for the null model over the main effects model $[85,86]$.

\subsubsection{Secondary outcomes}

We also asked participants directly if they would receive a COVID-19 vaccine if offered (yes/no). In the control group, $86.9 \%$ of participants responded 'yes'. A chi-squared test of independence indicated that the proportion of 'yes' responses did not differ significantly across message conditions $\left(X^{2}(4)=5.21, p=.27\right.$; Factbox: $84.4 \%$; Q\&A: 84.7\%; Approval: $87.6 \%$; Mechanism: $82.9 \%$ ).

While the mean level of side effect concern (measured by the adapted subscale of the Oxford COVID Vaccine Beliefs Scale) did not differ between groups, quantitative estimates of mild to moderate side effect frequency (per 10,000 vaccinations) differed between groups, $F(4,2080)=11.60, p<.001, \eta^{2}=0.02$. Post hoc tests indicated that the average estimate in the Factbox condition $(M=2663.86, S D=2922.01)$ was significantly higher than that in the control $(M=1782.92, S D=2515.07), Q \& A(M=1518.20, S D=2313.52)$, Approval $(M=1703.54, S D=2638.12)$, and Mechanism conditions $(M=1919.73, S D=2728.63$; all $p<$ $.001, d s$.26-.43). There was no main effect of condition on estimates of severe side effect 
frequency, $F(4,2083)=1.09, p=.36^{2}$, or estimates of vaccine efficacy (i.e. estimated percent of COVID-19 cases prevented): $\mathrm{F}(4,2089)=1.78, p=.13$ (across conditions, Ms 74.53$78.18 \%)$.

Lastly, we report a significant main effect of condition on responses to the 'informed' measure adapted from the Decisional Conflict Scale, $F(4,2091)=10.17, p<.001, \eta^{2}=0.02$ (see Figure 2). Post-hoc analyses revealed that participants in the Factbox condition $(M=$ $4.21, S D=0.84)$ reported, on average, feeling more informed regarding their vaccination decision that those in the control condition $(M=3.94, S D=0.91, p<.001, d=.30)$ and Mechanism condition $(M=4.04, S D=0.89, p=.04, d=.20$. Participants in the Q\&A condition $(M=4.28, S D=0.86)$ also reported feeling more informed than those in the control $(p<.001, d=.38)$, Mechanism $(p<.001, d=.28)$, and Approval conditions $(M=4.05, S D=$ $0.86, p=.001, d=.27)$. There was no effect of condition on decision certainty, $F(4,2092)=$ $0.53, p=.71$.

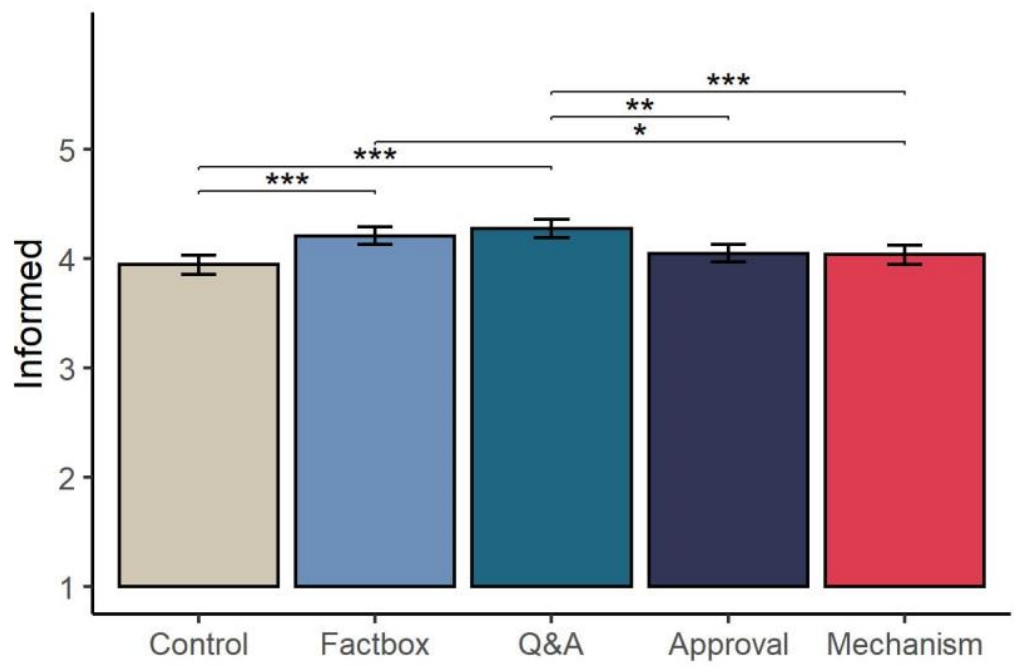

Figure 2: Mean $(95 \% \mathrm{CI})$ reported level of feeling informed regarding vaccination decision across experimental conditions. Brackets and asterisks indicate significant pairwise differences. ${ }^{*} p<.05$, ${ }^{* *} p<.01,{ }^{* * *} p<.001$.

Due to space, results of analyses focusing on ratings of the information (e.g. trustworthiness, believability, ease of understanding) are reported in the supplementary material (Appendix 3).

\subsection{Interim discussion}

Detailed information on any of: the efficacy and side effects of a COVID-19 vaccine (in Factbox or Q\&A format), regulatory process, or mechanism of vaccine action did not have any impact on vaccine beliefs or intentions. None of the messages in our experiment resulted in a shift (relative to control) in perceptions of efficacy, concerns over side-effects, concerns over the speed of regulatory approval or willingness to receive a vaccine.

The Factbox message, which stated the incidence of a number of side effects in a large clinical trial, did lead participants to give higher estimates of mild side effect frequency

2 As estimates of severe side effect frequency displayed notable skew ( $>4$ across all conditions), we also conducted nonparametric tests. We note this is a deviation from our pre-registered analyses. A Kruskal-Wallis test indicated a significant effect of condition on estimates, $H(4)=11.11, p=.02$. Post-hoc comparisons using Dunn's Test indicated that the median estimate in the Approval condition (Median $=20$ ) was significantly lower than the median estimate in the Factbox condition (Median $=50 ; p=.02$ ), but no message condition differed significantly from the control group. 
compared to the other conditions. Despite this increase, there was no corresponding increase in concern, suggesting that participants did not place much weight on mild side effects when it comes to weighing up the potential harms and benefits of vaccination against COVID-19. Such a conclusion aligns with the findings of a recent conjoint analysis of the effects of hypothetical COVID-19 vaccine attributes on acceptance. Kaplan and Milstein [53] report that varying rates of minor side-effects (between 50\% and 90\% likelihood) had no impact on participants willingness to receive a vaccine.

We note that the Factbox and the Q\&A messages did not have a significant impact on perceived efficacy. This is despite both messages detailing a high level of vaccine efficacy $(95.5 \%$ and $94.1 \%$ respectively), substantially higher than participants' average estimated level of efficacy as a percentage of cases prevented (across conditions, Ms 75-78\%). While this might suggest that people were not convinced by the information presented it may well also be the case that participants were adjusting their estimates of efficacy according to the quality of the evidence as they perceived it. The question did not ask 'what level of efficacy did the information provided say the vaccine has?', and so it is possible they indicating how well they thought the vaccines would actually perform in the real world. Such levels of adjustment of efficacy according to perceived quality of evidence have been seen in similar experiments around COVID-19 mitigations [78].

While none of the information had a significant effect on vaccine intentions, some messages increased participants reported awareness of the risks and benefits. Participants who read the Factbox or Q\&A materials scored higher on our subjective measure of being informed (i.e. reporting knowing the risks and benefits and feeling informed regarding their vaccination decision) relative to those in the control group. From an ethical standpoint, this improvement in understanding of the potential benefits and harms of vaccination is important, signalling a move towards better informed decision-making on behalf of the participants.

\section{Study 2}

While Study 1 focussed on the effect of messages about COVID-19 vaccines on vaccination attitudes and intentions, Study 2 focussed on a key question regarding the impact of uncertainty over vaccine efficacy and the need for vaccinated individuals to continue engaging in protective behaviours on both vaccine attitudes and intentions to carry out protective behaviour after vaccination [64].

Specifically, the study addressed two research questions: a) do messages that explicitly caveat the efficacy of vaccines (for self and others) affect perceptions of vaccine efficacy and vaccine intentions, and b) do they have an impact of intentions to follow recommendations post vaccination?

Six different messages were developed, modelled on existing communications from UK public health bodies, designed to vary greatly in the emphasis they put on uncertainty about the efficacy of vaccines and the need to continue protective behaviour once vaccinated. Our aim in presenting extremes of variation in emphasis was to explore the extent to which such messages could influence attitudes and behavioural intentions in an online survey experiment, rather than to identify the effects of particular aspects of such messaging (e.g. the potential protection of self versus others). This study was pre-registered (https://aspredicted.org/blind.php? $\mathrm{x}=\mathrm{r} 8 \mathrm{wp} 28$ ) and ethical oversight was provided by the Psychology Research Ethics Committee at the University of Cambridge PRE.2020.034 with amendment on $25^{\text {th }}$ January 2021).

\subsection{Methods}

\subsubsection{Participants}

We planned to compare two sets of messages (long and short; three of each, detailed below) with a control group separately. An a priori power calculation performed using GPOWER [72] indicated a total sample size of at least $N=1,724$ would be required to detect an effect size of $f=0.1$ in a one-way ANOVA with four groups, at 95\% power and 
an alpha level of .05. To account for the fact that our control group would be included in both sets of analyses, we doubled the size of the control group (thus the required sample size became $N=2155$ ). We deliberately recruited a larger sample to account for the exclusion criteria detailed below.

Participants were recruited through online panel provider Respondi (respondi.com) between 28 January-1 February 2021, and quota sampled to reflect the UK population in terms of age and sex. Individuals who did not provide informed consent, reported already having received a COVID-19 vaccination, or did not live in the UK were screened from taking the survey. A total of 2,509 participant's completed the survey. As per pre-registration, we excluded participants who failed an attention check ('please select somewhat agree'; $n=290)$, or provided an age outside of the range of $18-100$ years old $(n=2)$. This resulted in a final sample of 2,217 participants (47\% males; Mage $=46.33, S D=15.76 ; 88 \%$ White ethnicity; median highest education level: school education up to age 18).

\subsubsection{Materials and procedure}

After providing consent, participants were immediately randomised by the Qualtrics randomisation tool to one of seven conditions, to which they were blinded (with twice as many participants randomised to control as to each of the other conditions). Participants in the different information conditions (but not control) were told they would be shown 'a short piece of information about the COVID-19 vaccines' and asked to answer questions about it, including some testing recall. After completing the questions regarding COVID19 vaccines, all participants completed a series of demographic questions.

The six messages used in this study were modelled closely on publicly available communications available at the time of the study, including information pages from the Public Health England [87] website, and tweets from the UK NHS [65] and Public Health England [88] twitter accounts. We developed three types of message: No Caution, Medium Caution and High Caution. No caution messages highlighted the efficacy and safety of vaccines but made no mention of the need to continue protective behaviours once vaccinated. Medium Caution messages stated that while vaccines are broadly safe and effective, there is some uncertainty, and noted the need to maintain protective behaviours after vaccination. High Caution messages emphasized uncertainty around vaccine efficacy and noted the need to maintain protective behaviours after vaccination. For each type we created a Long version reflecting what might be found on a webpage, and a Short 2-3 sentence version formatted in the style of a social media post. All messages were presented with an identical image of vaccine vials. As an example, the Long of version of the Medium Caution message is shown in Figure 3. (Appendix 4 details the full text of all messages as presented to participants). 


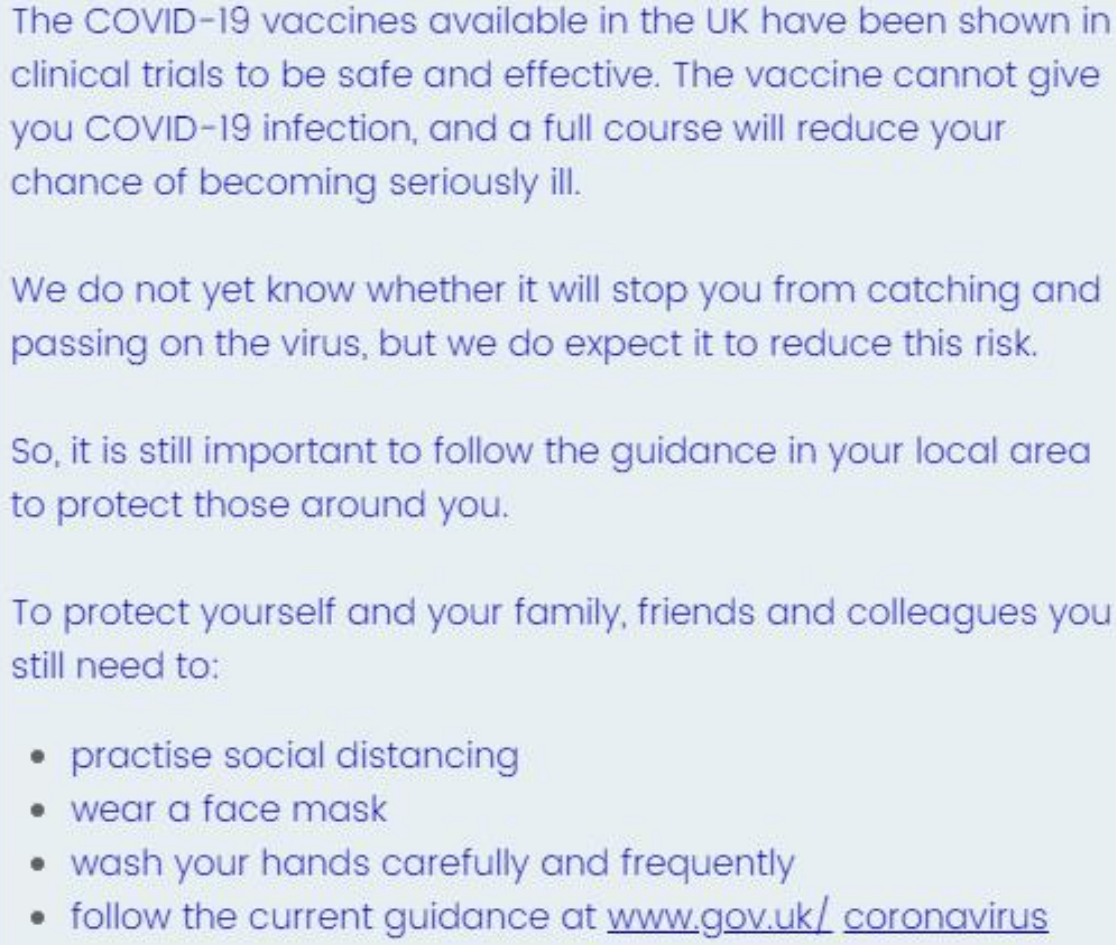

Figure 3: The Long Medium Caution message.

Participants then completed the following measures (in order presented; full item wording can be found in Appendix 5).

Primary Outcomes. Participants first completed the primary outcome measures. Intentions to engage in protective behaviour following vaccination were measured in two ways. First participants were asked to imagine they had been vaccinated and indicate how likely it is that they would engage in each of six behaviours once vaccinated (social distancing, frequent handwashing, wearing a facemask, avoiding social gatherings, working from home, avoiding public transport; 7pt response scale, Very unlikely to Very Likely). Responses were averaged to create a reliable index of protective behaviour intention $(\alpha=$ .86). Second, participants were asked if they would follow general rules post-vaccination, indicating their agreement with the statement: I would still follow whatever coronavirus rules or restrictions were in place as strictly as I was before getting a vaccine (7pt, Strongly disagree to Strongly agree). Willingness to receive a vaccine was measured using the Oxford COVID19 Vaccine Hesitancy Scale [12] ( $\alpha=.97$; as in Study 1, scores were reversed such that higher values indicate greater willingness).

Secondary Outcomes. Participants also completed a second, binary measure of vaccination intention, responding Yes or No to the question: 'If you were offered a COVID-19 vaccine would you get vaccinated yourself?' Hypothetical worry over COVID-19 risk behaviours post-vaccination was measured with a set of items in which participants indicated 
their level of worry regarding seven different behaviours (e.g. Shopping in a busy supermar$k e t)$ if they had already received the COVID-19 vaccine. Scores were averaged to create a single index $(\alpha=.95)$.

Participants who read a message (i.e. all groups other control) then completed several 'message only' measures relating to their impression of the information presented. Emotional response was captured using Marcus et al.'s [89] nine-item scale in which participants indicate how the information made them feel (e.g. Resentful) on a slider with the labels Not at all (0) and Extremely (100) and the slider initially anchored at 50. Following Marcus et al., responses were averaged to create three subscales: anxiety $(\alpha=.92)$, aversion $(\alpha=.93)$, and enthusiasm $(\alpha=.85)$. These participants also completed measures of: how trustworthy (three items, $\alpha=.97$ ), engaging (three items, $\alpha=.87$ ), and believable they found the information; how much they felt they understood the information; the level of effort required to read the information; and the perceived quality of the evidence underlying the information (all identical to Study 1).

All participants then completed the adapted version of the perceived efficacy $(\alpha=$ .84) and public importance (example: If I get the COVID-19 vaccine it will be: $1=$ Really helpful for the community around me, $5=$ Really unhelpful for the community around me; $\alpha=.84)$ subscales of the Oxford COVID-19 Vaccine Beliefs Scale, the informed $(\alpha=.81)$ and certainty ( $\alpha=.91$ ) measures adapted from the Decisional Conflict Scale, and provided estimates of the efficacy of the 'best available' COVID-19 vaccines (percent of cases prevented; all measures identical to Study 1).

\subsubsection{Analysis}

As per our preregistration, we compared outcome means between the long messages and control, and short messages and control separately using one-way ANOVAs (i.e. four groups in each separate analysis; hereafter differentiated by Long and Short subscripts in results).

\subsection{Results}

As in Study 1, participants, on average, considered the messages to be relatively trustworthy (Ms 5.46-5.71, SDs 1.28-1.47), engaging (Ms 5.47-5.76, SDs 1.20-1.30) and believable (Ms 5.41-5.66, SDs 1.29-1.46), with mean scores on these measures above the scale mid-point (range 1-7) across all message conditions (see Appendix 6 for comparisons between conditions).

\subsubsection{Primary outcomes}

Comparing both Long and Short messages separately with control, condition assignment was not significantly associated with willingness to receive a vaccine $\left(F_{\text {Long }}(3,1389)\right.$ $\left.=1.91, p=.13 ; F_{\text {Short }}(3,1396)=2.10, p=.10, \eta 2=0.00\right)$, intentions to follow general rules and restrictions $\left(F_{\operatorname{Long}}(3,1390)=1.38, p=.25 ; F_{\text {Short }}(3,1396)=0.66, p=.57\right)$, or to engage in specific behaviours following vaccination $\left(F_{\operatorname{Long}}(3,1390)=2.28, p=.08 ; F_{\text {Short }}(3,1395)=0.95, p=.42\right)$. See Figure 4. 
A

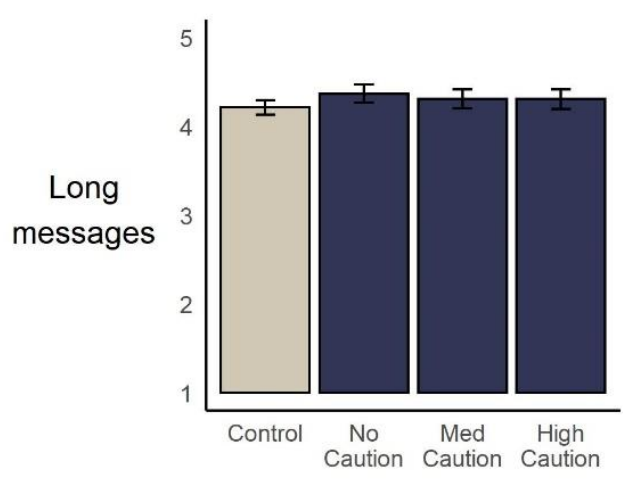

B

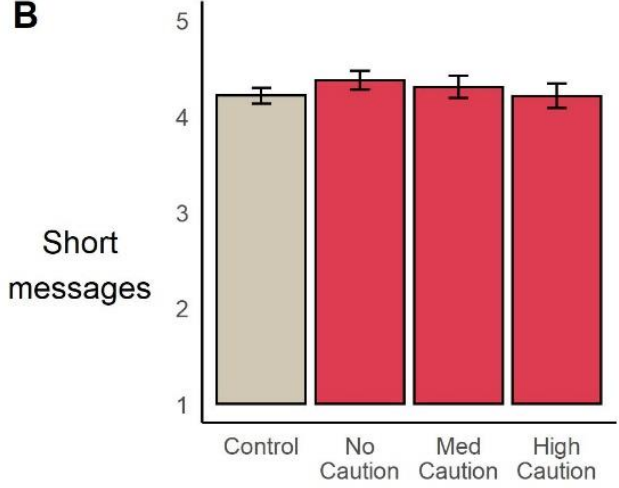

Vaccine intentions
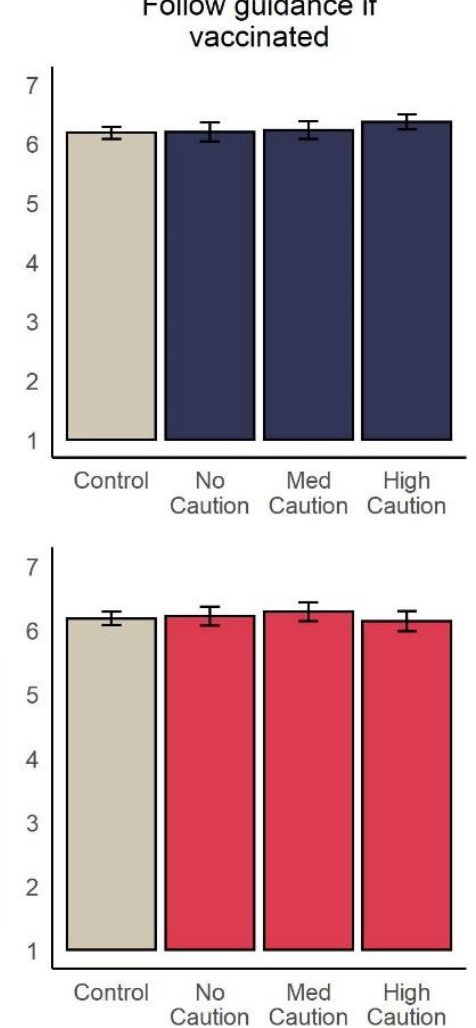

Content
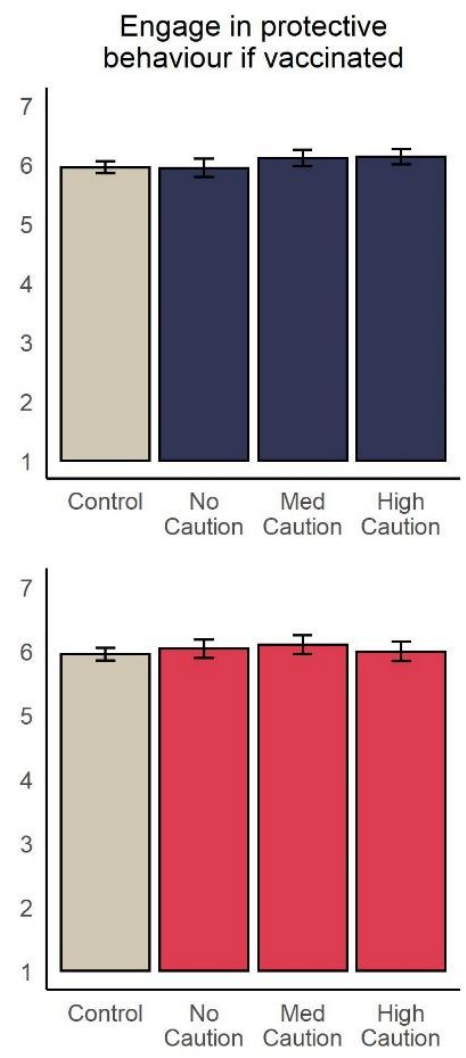

Figure 4: Mean $(95 \% \mathrm{CI})$ levels of vaccine and behavioural intentions, across experimental conditions. In line with ANOVAs reported in main text, Long messages (A) and Short messages (B) are separately compared to control.

To examine extent to which the data supports a null effect model, we conducted Bayesian ANOVAs. Resulting Bayes factors indicated 'substantial' to 'very strong' evidence for null models in which message condition has no effect vaccine intentions, ( $B F$ Long $\left.=30.46 ; B F_{\text {short }}=24.13\right)$, intentions to follow rules $\left(B F_{\text {Long }}=67.66 ; B F_{\text {short }}=190.01\right)$ or to engage in protective behaviour post vaccination $\left(B F_{\text {Long }}=18.38 ; B F_{\text {short }}=125.14\right)$.

\subsubsection{Secondary outcomes}

As in Study 1, we included an alternative binary (yes/no) measure of vaccine intentions in the experiment. In the control group, $84.9 \%$ of participants indicated they would receive a vaccine if available. A chi-squared test of independence indicated that the proportion of 'yes' responses did not differ significantly across message conditions $\left(X^{2}(6)=\right.$ $4.78, p=.57)$. Additional tests comparing only Long messages and the control group $\left(X^{2}(3)=3.55, p=.31\right)$ and Short messages and the control group $\left(X^{2}(3)=2.08, p=.56\right)$ were also non-significant.

We report no main effect of message condition on reported worry over risk behaviours if vaccinated $\left(F_{\text {Long }}(3,1390)=2.46, p=.06 ; F_{\text {Short }}(3,1396)=0.72, p=.54\right)$, the perceived public importance of vaccines $\left(F_{\text {Long }}(3,1390)=1.87, p=.13\right.$; $\left.F_{\text {short }}(3,1396)=2.16, p=.09\right)$, or decision certainty $\left(F_{\text {Long }}(3,1388)=2.44, p=.06 ; F_{\text {short }}(3,1394)=1.87, p=.13\right)$.

Comparing between Long messages and the control group, we do find a significant effect of message on perceived efficacy of vaccines, $F(3,1389)=7.76, p<.001, \eta^{2}=0.02$. Post hoc tests revealed that participants in the No Caution condition $(M=4.14, S D=0.70)$ perceived COVID-19 vaccines to be more effective than participants in the control $(M=3.89$, $S D=0.77 ; p<.001, d=.34)$, Medium Caution $(M=3.93, S D=0.71 ; p<.01, d=.29)$, and High Caution $(M=3.92, S D=0.73 ; p<.01, d=.31)$ conditions. There was a significant effect of 
condition on quantitative estimates of vaccine efficacy (i.e. estimated percent of cases prevented by vaccine), $F(3,1386)=8.09 ; p<.001, \eta 2=0.02$, with the mean estimate in the No caution condition $(M=78.65 \%, S D=20.76)$ significantly higher than that in the control ( $M$ $=70.99 \%, S D=23.07 ; p<.001, d=.35)$ and Medium Caution $(M=73.75 \%, S D=19.97, p=$ $.04, d=.24)$ conditions. We also note a significant effect of condition on participants' reported level of feeling informed regarding their vaccination decision, $\mathrm{F}(3,1390)=4.96, p<$ $.01, \eta^{2}=0.01$, with average responses in the No Caution $(M=4.20, S D=0.83, p=.02, d=$ $.22)$ and Medium Caution $(M=4.22, S D=0.81 ; p=.006, d=.25)$ conditions higher than that in control condition $(M=4.01, S D=0.93)$, as shown in Figure 5.
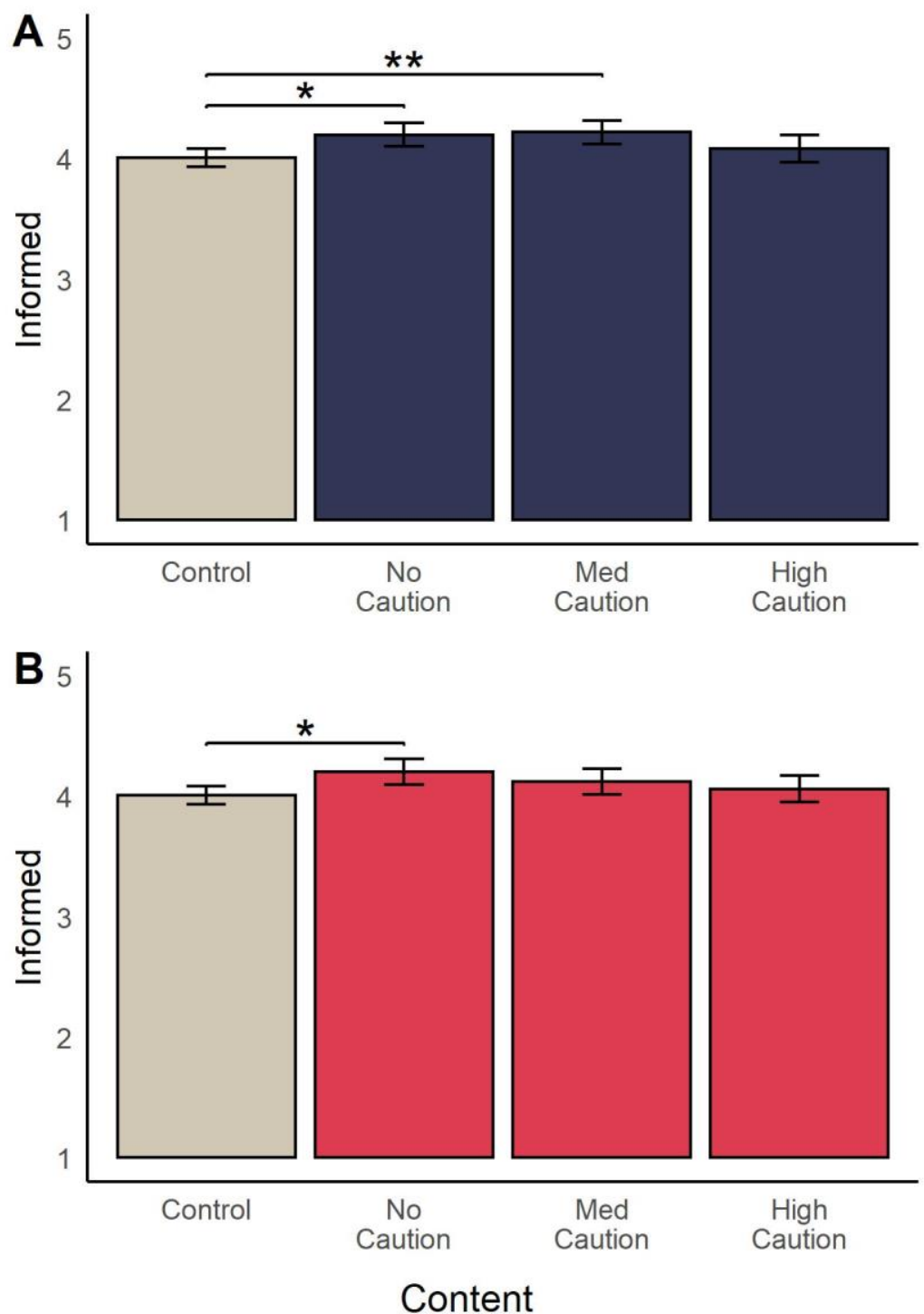

Figure 5: Mean (95\%CI) reported level of feeling informed after reading (A) Long and (B) Short messages. Brackets and asterisks indicate significant pairwise differences between conditions. ${ }^{*} p<$ $.05,{ }^{* *} p<.01$.

Comparing Short messages and the control, we also report a significant effect of condition on perceived efficacy $(F(3,1394)=4.86, p<.01, \eta 2=0.01)$ and estimated efficacy $\left(\mathrm{F}(3,1392)=8.59, p<.001, \eta^{2}=0.02\right)$. Post hoc tests indicated that perceived efficacy was significantly higher in the No Caution condition $(M=4.05, S D=0.72)$ compared to the control $(M=3.89, S D=0.77 ; p=.02, d=.21$ and High Caution conditions $(M=3.86, S D=$ $0.83 ; p=.03, d=.23$ ). For quantitative estimates of vaccine efficacy, we find that estimates 
in the No Caution condition $(M=76.10 \%, S D=20.00)$ were higher than those in the control condition $(M=70.99 \%, S D=23.07 ; p<.01, d=.24)$, as were estimates in the Medium Caution condition $(M=78.69 \%, S D=20.95 ; p<.001, d=.35)$. The ANOVA test of the effect of condition on feeling informed was significant, $F(3,1396)=3.09, p=.03, \eta^{2}=0.01$ : participants' in the Caution condition $(M=4.20, S D=0.92)$ reported feeling more informed than those in the control condition $(M=4.01, S D=0.93 ; p=.02, d=.21$; Figure 5$)$.

There were a number of measures relating to perceptions of the information shown which were not presented to participants in the control condition (because these participants did not see any message). For these outcomes, we conducted 2(length)x3(content) ANOVAs to examine the effect of both message length and content. Briefly, we find there was no significant effect of either factor, or interaction between them, on: perceived trustworthiness of the information; perceived quality evidence upon which the message was based; how believable the information was; how difficult the information was to understand. Longer messages were considered more engaging than short messages. Considering emotional responses to messages, No Caution messages elicited more enthusiasm and less aversion and anxiety compared to other conditions (full results reported in Appendix $6)$.

\subsection{Study 2 discussion}

As in Study 1, we find that intentions to vaccinate remain relatively static in the face of messages about vaccine efficacy and safety. We also find that the inclusion of uncertainty and reminders to maintain protective behaviours once vaccinated do not have a substantial effect on intentions to vaccinate, or on intentions to engage in protective behaviours if vaccinated. This will be of some comfort to public health communicators concerned that calls to continue social distancing and mask-wearing once vaccinated - another important public health message [64] - might undermine perceptions of vaccine efficacy.

Considering our secondary outcomes, we did find that positive (i.e. No Caution) messages did increase the subjective perceived efficacy of the vaccines and quantitative estimates of vaccine efficacy, relative to the control group. Similarly, both Long and Short No caution messages (and the Long Medium caution message) increased participants' sense of feeling informed regarding their vaccination decision compared to the control group. This is an interesting comparison with Study 1, where it was the provision of more informative messages that increased reported informedness. Here in Study 2 it is the least informative messages that increased the subjective impression of being informed. This raises the issue of self-report knowledge appraisals not being a good measure of decision quality when taken in isolation: a decision made easy through simplified or persuasive messaging, which might lead to high rating on decision confidence, is not necessarily a good decision.

\section{General discussion}

The clear and transparent communication of COVID-19 vaccines' risks and benefits has been suggested as an approach to increasing vaccine uptake among the general public $[14,16]$, a means of maintaining public trust in science (e.g., $[15,21])$, and also as an ethical imperative. We investigated how communications about the efficacy and side effects of COVID-19 vaccines affect individuals' vaccination intentions and related beliefs. Across two experiments we find that intention to receive a vaccine when offered is relatively high and that different messages - ranging from detailed tables of vaccine efficacy and side effects to a simple claim that vaccines are safe and effective - do not substantially increase intentions to receive a COVID-19 vaccine. Encouragingly, we also did not find any evidence that such communications lead to a decrease in vaccination intentions either, such as through 'backfire effects' [90]. This was true even for messages in Study 2 which expressed high uncertainty about vaccine efficacy (a range of "41-98\%") and reminded the reader that protective behaviours would need to be maintained even once vaccinated. 
We also examined how messages influenced participants' perceptions of COVID19 vaccine efficacy and safety, beliefs which are consistently associated with vaccine intentions $[8,12]$. These beliefs remained essentially unchanged in the face of detailed information from clinical trials. We do note that in Study 2, simple claims that vaccines are safe and effective (with or without reference to specific details; i.e. both short and long versions) did increase perceptions of efficacy relative to controls, but had no main effect on vaccine intentions. This gives some indirect evidence that increasing perceptions of vaccine efficacy may have a limited subsequent impact on intentions. However further research should investigate the causal effects of changes in perceived efficacy on intentions to confirm this.

Taken as whole, our main results align with the wider research on non-COVID-19 vaccine messaging, which indicates that simple written or visual purely informational messages have limited effects on vaccine hesitancy and uptake [91,92]. Our results also indicate that COVID-19 vaccine hesitancy is not simply due to "a deficit of the data needed to make an informed decision," as suggested by Opel et al., [29]. While the current studies do not provide a strong basis for public health authorities to communicate vaccine information with the aim of increasing uptake of the vaccine, we would note two important points: first, our study occurred in the midst of such communications, and second, increasing uptake is not the only reason for actively disseminating vaccine evidence such as the results of clinical trials.

On the first point, public health bodies in the UK, where these studies were carried out, have been active in communicating information about COVID-19 vaccines as it becomes available, and such information has received intense media attention. This may have already contributed to the high levels of vaccination intentions. As such, we may be constrained by a celling effect in the current study. We did not measure attitudes prior to the experimental interventions, but it is possible that there was a greater effect of information on people who were opposed to or undecided regarding COVID-19 vaccination. Future research could investigate such moderation effects, incorporating prior beliefs into experimental models or using pre-post within subjects designs.

Regarding the second point, governments and responsible public health bodies have an ethical duty to inform the public of the risks and benefits of the vaccines they are asking the public to take [93]. Thus, informing the public, should be considered an important endpoint in itself. We did find some positive results in this regard; in Study 1 several of the more informative messages increased participants' sense of feeling informed regarding the decision of whether or not to vaccinate. Such transparent communication may also serve to build trust over the longer term [94,95].

One notable omission from the various the framings included in this study was a focus on prosocial or collective benefits of vaccination. At the time of data collection, there was no peer-reviewed research indicating that COVID-19 vaccines could prevent transmission of the virus. As such, we did not include any messages detailing how the choice to vaccinate might protect others. Previous research on hesitancy towards other vaccines $[96,97]$ and preliminary studies on COVID-19 vaccine intentions $[43,54]$ suggest that this framing may be effective in increasing vaccine acceptance. As more evidence of the effect of vaccination on transmission becomes available, researchers should investigate the how communicating such evidence impacts vaccine attitudes and intentions. This may be especially relevant for increasing vaccination rates in younger age groups who are at much lower risk of death or hospitalisation from COVID-19 and perceive less threat from the virus $[31,98]$. Another factor we did not examine in the current studies was the impact of message source. It is possible that attributing the information to a source considered trustworthy by the UK public (e.g. the NHS) may have increased the impact of information.

Beyond broad messaging approaches, those seeking to increase vaccine uptake have a range of tools at their disposal. Interventions such as messaging targeted specifically to hesitant groups, working with trusted community leaders, training healthcare worker for face-to-face conversations with vaccine hesitant individuals, and reducing financial or 
logistical barriers (e.g. access to vaccination sites) have all been shown to increase vaccine uptake in some contexts (for reviews see: $[9,91,99]$ ). A comprehensive approach to addressing COVID-19 vaccine hesitancy should draw on all available tools, and not rely solely on communicating vaccine information via messages like those examined in the current studies.

We must note some limitations to the current studies. Both used online panel samples, which may not be reflective of the wider UK population. However, we would note that our samples were matched to the UK population in terms of age and gender, and the proportion of participants willing to receive a vaccine was in line with weighted population estimates reported in a contemporaneous YouGov survey [88.8\% in February 2021; ,100]. Readers should also be mindful that results from our UK studies may not generalise to other countries, as many local contextual factors can influence vaccine hesitancy [9]. For example, the development of the Oxford AstraZeneca vaccine has been hailed by the media as a 'British' success story [e.g., 101] and this may lead to pro-vaccine attitudes being linked with national identity in the UK.

In conclusion, we find that messages outlining the known risks and benefits of COVID-19 vaccines (with varying levels of detail) have little impact on vaccination intentions or beliefs about COVID-19 vaccines in UK online samples but some messages increase reported understanding of the risk and benefits. We also found no evidence that emphasising the need for continuing protective behaviours post-vaccination undermined vaccination intentions or perceptions of efficacy. These results indicate that there are no substantial effects of transparent communication on reducing vaccination intentions. However, they also highlight the need for those who wish to increase vaccination intentions or protective behaviours post vaccination to draw on a range of strategies - not just transparent communication of risk and benefits. 
Supplementary Materials: The following are available online at www.mdpi.com/xxx/s1: Appendices 1-6.

Author Contributions: Conceptualization, J.K., A.F., S.vdL. and T.M.; investigation, J.K., A.F., S.vdL. and T.M.; data curation, J.K.; analysis, J.K.; writing-original draft preparation, J.K.; writing - review and editing, J.K., A.F., S.vdL. and T.M.; visualization, X.X.; supervision, A.F., S.vdL . and T.M.. All authors have read and agreed to the published version of the manuscript.

Funding: This study was funded by the Winton Centre for Risk and Evidence Communication which is supported by the David and Claudia Harding Foundation.

Institutional Review Board Statement: The study was conducted according to the guidelines of the Declaration of Helsinki, and ethical oversight was provided by the Psychology Research Ethics Committee at the University of Cambridge (PRE.2020.034, amendments approved $9^{\text {th }}$ and $25^{\text {th }}$ January, 2021).

Informed Consent Statement: Informed consent was obtained from all subjects involved in the study.

Data Availability Statement: Data and analysis code for these studies are available at: https://osf.io/hsugy/?view only=16fbf1f5737a43db8f9d20a7b2d3db4b

Acknowledgments: We would like to thank Lisa-Maria Tanase for assistance in developing and proofing survey materials.

Conflicts of Interest: The authors declare no conflict of interest.

\section{References}

1. WHO Weekly epidemiological update - 9 March 2021 Available online: https://www.who.int/publications/m/item/weeklyepidemiological-update---10-march-2021 (accessed on Mar 12, 2021).

2. Saladino, V.; Algeri, D.; Auriemma, V. The Psychological and Social Impact of Covid-19: New Perspectives of Well-Being. Front. Psychol. 2020, 11, 2550, doi:10.3389/fpsyg.2020.577684.

3. Chowdhury, E.K.; Khan, I.I.; Dhar, B.K. Catastrophic impact of Covid-19 on the global stock markets and economic activities. Bus. Soc. Rev. 2021, basr.12219, doi:10.1111/basr.12219.

4. Pearl, R. The Queen's Gambit And Covid-19: Why It's All About The End Game Now Available online: https://www.forbes.com/sites/robertpearl/2020/12/07/the-queens-gambit-and-covid-19-its-all-about-the-end-gamenow/?sh=1cc0447d3f8a (accessed on Mar 3, 2021).

5. Randolph, H.E.; Barreiro, L.B. Herd Immunity: Understanding COVID-19. Immunity 2020, 52, 737-741.

6. Anderson, R.M.; Vegvari, C.; Truscott, J.; Collyer, B.S. Challenges in creating herd immunity to SARS-CoV-2 infection by mass vaccination. Lancet 2020, 396, 1614-1616.

7. Kadkhoda, K. Herd Immunity to COVID-19. Am. J. Clin. Pathol. 2021, XX, 1-2, doi:10.1093/ajcp/aqaa272.

8. Lin, C.; Tu, P.; Beitsch, L.M. Confidence and receptivity for covid-19 vaccines: A rapid systematic review. Vaccines 2021.

9. WHO Report of the Sage Working Group on Vaccine Hesitancy; 2014;

10. de Figueiredo, A.; Simas, C.; Karafillakis, E.; Paterson, P.; Larson, H.J. Mapping global trends in vaccine confidence and investigating barriers to vaccine uptake: a large-scale retrospective temporal modelling study. Lancet 2020, 396, 898-908, doi:10.1016/S0140-6736(20)31558-0.

11. Kerr, J.R.; Schneider, C.R.; Recchia, G.; Dryhurst, S.; Sahlin, U.; Dufouil, C.; Arwidson, P.; Freeman, A.L.J.; van der Linden, S. Predictors of COVID-19 vaccine acceptance across time and countries. medRxiv 2020, doi:10.1101/2020.12.09.20246439.

12. Freeman, D.; Loe, B.S.; Chadwick, A.; Vaccari, C.; Waite, F.; Rosebrock, L.; Jenner, L.; Petit, A.; Lewandowsky, S.; Vanderslott, S.; et al. COVID-19 vaccine hesitancy in the UK: The Oxford coronavirus explanations, attitudes, and narratives survey (Oceans) II. Psychol. Med. 2021, 1-15, doi:10.1017/S0033291720005188.

13. Quinn, S.C.; Jamison, A.M.; Freimuth, V. Communicating Effectively About Emergency Use Authorization and Vaccines in 
the COVID-19 Pandemic. Am. J. Public Health 2021, 111, 355-358, doi:10.2105/ajph.2020.306036.

14. Cohen, A.F.; van Gerven, J.; Burgos, J.G.; de Boer, A.; Foucher, R.A.M.; Flore, H.; Teitelbaum, Z.; van Eden, W.; Webb, A.; Cremers, S. COVID-19 vaccines: the importance of transparency and fact-based education. Br. J. Clin. Pharmacol. 2020, 86, 2107-2110.

15. Nature COVID vaccine confidence requires radical transparency. Nature 2020, 586, 8, doi:10.1038/d41586-020-02738-y.

16. Rzymski, P.; Borkowski, L.; Drąg, M.; Flisiak, R.; Jemielity, J.; Krajewski, J.; Mastalerz-Migas, A.; Matyja, A.; Pyrć, K.; Simon, K.; et al. The Strategies to Support the COVID-19 Vaccination with Evidence-Based Communication and Tackling Misinformation. Vaccines 2021, 9, 109, doi:10.3390/vaccines9020109.

17. Blastland, M.; Freeman, A.L.J.; van der Linden, S.; Marteau, T.M.; Spiegelhalter, D. Five rules for evidence communication. Nature 2020, 587, 362-364, doi:10.1038/d41586-020-03189-1.

18. Cousins, S.; Blencowe, N.S.; Blazeby, J.M. What is an invasive procedure? A definition to inform study design, evidence synthesis and research tracking. BMJ Open 2019, 9, 2018-2020, doi:10.1136/bmjopen-2018-028576.

19. Reiss, D.R.; Karako-Eyal, N. Informed Consent to Vaccination: Theoretical, Legal, and Empirical Insights. Am. J. Law Med. 2019, 45, 357-419, doi:10.1177/0098858819892745.

20. O'Neill, O. Some limits of informed consent. Elder. Leg. Ethical Issues Healthc. Policy 2017, 103-106, doi:10.4324/97813152400469.

21. Parmet, W.E. Informed consent and public health: are they compatible when it comes to vaccines? J. Health Care Law Policy 2005, 8, 71-110.

O'Neill, J. Case for persuasion in parental informed consent to promote rational vaccine choices. J. Med. Ethics 2020, 1-6, doi:10.1136/medethics-2020-106068.

24. Marco-Franco, J.E.; Pita-Barros, P.; Vivas-Orts, D.; González-de-Julián, S.; Vivas-Consuelo, D. COVID-19, Fake News, and Vaccines: Should Regulation Be Implemented? Int. J. Environ. Res. Public Health 2021, 18, 744, doi:10.3390/ijerph18020744.

Petersen, M.B.; Bor, A.; Jørgensen, F.J.; Lindholt, M.F. Transparency Is Necessary but Not Sufficient to Reduce Skepticism About a COVID-19 Vaccine. PsyArXiv 2020, doi:10.31234/OSF.IO/VX84N.

26. Rossen, I.; Hurlstone, M.J.; Lawrence, C. Going with the Grain of Cognition: Applying Insights from Psychology to Build Support for Childhood Vaccination. Front. Psychol. 2016, 7, 1483, doi:10.3389/fpsyg.2016.01483.

Simis, M.J.; Madden, H.; Cacciatore, M.A.; Yeo, S.K. The lure of rationality: Why does the deficit model persist in science communication? Public Underst. Sci. 2016, 25, 400-414, doi:10.1177/0963662516629749.

28. Kitta, A.; Goldberg, D.S. The significance of folklore for vaccine policy: discarding the deficit model. Crit. Public Health 2017, $27,506-514$.

29. Opel, D.J.; Salmon, D.A.; Marcuse, E.K. Building Trust to Achieve Confidence in COVID-19 Vaccines. JAMA Netw. Open 2020, 3, e2025672-e2025672, doi:10.1001/jamanetworkopen.2020.25672.

30. Lin, Y.; Hu, Z.; Zhao, Q.; Alias, H.; Danaee, M.; Wong, L.P. Understanding COVID-19 vaccine demand and hesitancy: A nationwide online survey in China. PLoS Negl. Trop. Dis. 2020, doi:10.1371/journal.pntd.0008961.

31. Karlsson, L.C.; Soveri, A.; Lewandowsky, S.; Karlsson, L.; Karlsson, H.; Nolvi, S.; Karukivi, M.; Lindfelt, M.; Antfolk, J. Fearing the disease or the vaccine: The case of COVID-19. Pers. Individ. Dif. 2021, doi:10.1016/j.paid.2020.110590.

32. Wang, K.; Wong, E.L.Y.; Ho, K.F.; Cheung, A.W.L.; Yau, P.S.Y.; Dong, D.; Wong, S.Y.S.; Yeoh, E.K. Change of willingness to accept covid-19 vaccine and reasons of vaccine hesitancy of working people at different waves of local epidemic in hong kong, china: Repeated cross-sectional surveys. Vaccines 2021, doi:10.3390/vaccines9010062.

33. Callaghan, T.; Moghtaderi, A.; Lueck, J.A.; Hotez, P.; Strych, U.; Dor, A.; Fowler, E.F.; Motta, M. Correlates and disparities of intention to vaccinate against COVID-19. Soc. Sci. Med. 2021, doi:10.1016/j.socscimed.2020.113638. 
34. Paul, E.; Steptoe, A.; Fancourt, D. Attitudes towards vaccines and intention to vaccinate against COVID-19: Implications for public health communications. Lancet Reg. Heal. - Eur. 2021, 1, 100012, doi:10.1016/j.lanepe.2020.100012.

35. Wong, L.P.; Alias, H.; Wong, P.-F.; Lee, H.Y.; AbuBakar, S. The use of the health belief model to assess predictors of intent to receive the COVID-19 vaccine and willingness to pay. Hum. Vaccin. Immunother. 2020, 16, 2204-2214, doi:10.1080/21645515.2020.1790279.

36. Fisher, K.A.; Bloomstone, S.J.; Walder, J.; Crawford, S.; Fouayzi, H.; Mazor, K.M. Attitudes Toward a Potential SARS-CoV-2 Vaccine: A Survey of U.S. Adults. Ann. Intern. Med. 2020, doi:10.7326/M20-3569.

37. Frank, K.; Arim, R. Canadians' willingness to get a COVID-19 vaccine when one becomes available: What role does trust play? Available online: https://www150.statcan.gc.ca/n1/pub/45-28-0001/2020001/article/00043-eng.htm (accessed on Nov 22, 2020).

38. Yoda, T.; Katsuyama, H. Willingness to receive covid-19 vaccination in Japan. Vaccines 2021, doi:10.3390/vaccines9010048.

39. Ruiz, J.B.; Bell, R.A. Predictors of intention to vaccinate against COVID-19: Results of a nationwide survey. Vaccine 2021, doi:10.1016/j.vaccine.2021.01.010.

40. Yigit, M.; Ozkaya-Parlakay, A.; Senel, E. Evaluation of COVID-19 Vaccine Refusal in Parents. Pediatr. Infect. Dis. J. 2021, doi:10.1097/inf.0000000000003042.

41. Momplaisir, F.; Haynes, N.; Nkwihoreze, H.; Nelson, M.; Werner, R.M.; Jemmott, J. Understanding Drivers of COVID-19 Vaccine Hesitancy Among Blacks. Clin. Infect. Dis. 2021, doi:10.1093/cid/ciab102.

42. Lockyer, B.; Islam, S.; Rahman, A.; Dickerson, J.; Pickett, K.; Sheldon, T.; Wright, J.; McEachan, R.; Sheard, L. Understanding Covid-19 misinformation and vaccine hesitancy in context: Findings from a qualitative study involving citizens in Bradford, UK. medRxiv 2020.

43. Williams, L.; Gallant, A.J.; Rasmussen, S.; Brown Nicholls, L.A.; Cogan, N.; Deakin, K.; Young, D.; Flowers, P. Towards intervention development to increase the uptake of COVID-19 vaccination among those at high risk: Outlining evidencebased and theoretically informed future intervention content. Br. J. Health Psychol. 2020, 25, 1039-1054, doi:10.1111/bjhp.12468.

44. Hotez, P.J. COVID19 meets the antivaccine movement. Microbes Infect. 2020, 22, 162-164.

45. Roozenbeek, J.; Schneider, C.R.; Dryhurst, S.; Kerr, J.; Freeman, A.L.J.; Recchia, G.; van der Bles, A.M.; van der Linden, S. Susceptibility to misinformation about COVID-19 around the world. R. Soc. Open Sci. 2020, 7, 201199, doi:10.1098/rsos.201199.

46. Carpenter, C.J. A Meta-Analysis of the Effectiveness of Health Belief Model Variables in Predicting Behavior. Health Commun. 2010, 25, 661-669, doi:10.1080/10410236.2010.521906.

47. Maiman, L.A.; Becker, M.H. The Health Belief Model: Origins and Correlates in Psychological Theory. Health Educ. Monogr. 1974, 2, 336-353, doi:10.1177/109019817400200404.

48. Jones, C.L.; Jensen, J.D.; Scherr, C.L.; Brown, N.R.; Christy, K.; Weaver, J. The Health Belief Model as an Explanatory Framework in Communication Research: Exploring Parallel, Serial, and Moderated Mediation. Health Commun. 2015, 30, 566576, doi:10.1080/10410236.2013.873363.

49. Motta, M. Can a COVID-19 vaccine live up to Americans' expectations? A conjoint analysis of how vaccine characteristics influence vaccination intentions. Soc. Sci. Med. 2021, doi:10.1016/j.socscimed.2020.113642.

50. Borriello, A.; Master, D.; Pellegrini, A.; Rose, J.M. Preferences for a COVID-19 vaccine in Australia. Vaccine 2021, doi:10.1016/j.vaccine.2020.12.032.

51. Kreps, S.; Prasad, S.; Brownstein, J.S.; Hswen, Y.; Garibaldi, B.T.; Zhang, B.; Kriner, D.L. Factors Associated With US Adults' Likelihood of Accepting COVID-19 Vaccination. JAMA Netw. open 2020, 3, e2025594, doi:10.1001/jamanetworkopen.2020.25594.

52. Schwarzinger, M.; Watson, V.; Arwidson, P.; Alla, F.; Luchini, S. COVID-19 vaccine hesitancy in a representative workingage population in France: a survey experiment based on vaccine characteristics. Lancet Public Heal. 2021, 0, doi:10.1016/S2468- 
2667(21)00012-8.

53. Kaplan, R.M.; Milstein, A. Influence of a COVID-19 vaccine's effectiveness and safety profile on vaccination acceptance. Proc. Natl. Acad. Sci. U. S. A. 2021, 118, 2021726118, doi:10.1073/pnas.2021726118.

54. Motta, M.; Sylvester, S.; Callaghan, T.; Lunz-Trujillo, K. Encouraging COVID-19 Vaccine Uptake Through Effective Health Communication. Front. Polit. Sci. 2021, 3, 1, doi:10.3389/fpos.2021.630133.

55. Palm, R.; Bolsen, T.; Kingsland, J.; Alakaloko, F.M.; Akase, I.E.; Otrofanowei, E.; Salu, O.B.; Adeyemo, W.L.; Ademuyiwa, A.O.; Omilabu, S.A.; et al. The Effect of Frames on COVID-19 Vaccine Hesitancy. medRxiv 2021.

56. Loomba, S.; de Figueiredo, A.; Piatek, S.J.; de Graaf, K.; Larson, H.J. Measuring the impact of COVID-19 vaccine misinformation on vaccination intent in the UK and USA. Nat. Hum. Behav. 2021, 1-12, doi:10.1038/s41562-021-01056-1.

57. Duquette, N. Heard Immunity: Effective Persuasion for a Future COVID-19 Vaccine. SSRN Electron. J. 2020, doi:10.2139/ssrn.3705504.

58. Altay, S.; Hacquin, A.-S.; Chevallier, C.; Mercier, H. Information Delivered by a Chatbot Has a Positive Impact on COVID19 Vaccines Attitudes and Intentions. PsyArXiv 2021, doi:10.31234/OSF.IO/EB2GT.

59. Ranney, M.A.; Clark, D. Climate change conceptual change: Scientific information can transform attitudes. Top. Cogn. Sci. 2016, 8, 49-75, doi:10.1111/tops.12187.

60. McPhetres, J.; Rutjens, B.T.; Weinstein, N.; Brisson, J.A. Modifying attitudes about modified foods: Increased knowledge leads to more positive attitudes. J. Environ. Psychol. 2019, 64, 21-29, doi:10.1016/J.JENVP.2019.04.012.

61. Baden, L.R.; El Sahly, H.M.; Essink, B.; Kotloff, K.; Frey, S.; Novak, R.; Diemert, D.; Spector, S.A.; Rouphael, N.; Creech, C.B.; et al. Efficacy and Safety of the mRNA-1273 SARS-CoV-2 Vaccine. N. Engl. J. Med. 2021, 384, 403-416, doi:10.1056/NEJMoa2035389.

62. Torjesen, I. Covid-19: AstraZeneca vaccine is approved in EU with no upper age limit. BMJ 2021, 372, n295, doi:10.1136/bmj.n295.

63. The Lancet Microbe COVID-19 vaccines: the pandemic will not end overnight. The Lancet Microbe 2021, 2, e1, doi:10.1016/s2666-5247(20)30226-3.

64. Su, Z.; Wen, J.; McDonnell, D.; Goh, E.; Li, X.; Šegalo, S.; Ahmad, J.; Cheshmehzangi, A.; Xiang, Y.-T. Vaccines are not yet a silver bullet: The imperative of continued communication about the importance of COVID-19 safety measures. Brain, Behav. Immun. - Heal. 2021, doi:10.1016/j.bbih.2021.100204.

65. NHS If you've had the COVID-19 vaccine, it's vital that you continue to follow social distancing guidance. Read the latest guidance here [tweet] 2021.

66. CDC What to Expect after Getting a COVID-19 Vaccine [Archived webpage] Available online: https://web.archive.org/web/20210228020728/https:/www.cdc.gov/coronavirus/2019-ncov/vaccines/expect/after.html (accessed on Mar 2, 2021).

67. Pennings, S.; Symons, X. Persuasion, not coercion or incentivisation, is the best means of promoting COVID-19 vaccination. J. Med. Ethics 2021, doi:10.1136/medethics-2020-107076.

68. Prasad, V. Op-Ed: Throw Away Your Mask After COVID Vaccination? Available online: https://www.medpagetoday.com/blogs/vinay-prasad/90764 (accessed on Mar 17, 2021).

69. Krupnikov, Y.; Findley, B. Survey experiments: Managing the methodological costs and benefits. In The Oxford Handbook of Polling and Polling Methods; Oxford University Press, 2015; pp. 483-504 ISBN 9780190213299.

70. Nyhan, B.; Reifler, J. Does correcting myths about the flu vaccine work? An experimental evaluation of the effects of corrective information. Vaccine 2015, 33, 459-464, doi:10.1016/j.vaccine.2014.11.017.

71. Godinho, C.A.; Yardley, L.; Marcu, A.; Mowbray, F.; Beard, E.; Michie, S. Increasing the intent to receive a pandemic influenza vaccination: Testing the impact of theory-based messages. Prev. Med. (Baltim). 2016, 89, 104-111, 
doi:10.1016/j.ypmed.2016.05.025.

72. Erdfelder, E.; Faul, F.; Buchner, A. GPOWER: A general power analysis program. Behav. Res. Methods, Instruments, Comput. 1996, 28, 1-11, doi:10.3758/BF03203630.

73. Göritz, A.S. Using Online Panels in Psychological Research. In Oxford Handbook of Internet Psychology; Oxford University Press, 2012 ISBN 9780191743771.

74. Prolific Representative Samples on Prolific Available online: https://researcher-help.prolific.co/hc/engb/articles/360019236753-Representative-Samples-on-Prolific (accessed on Oct 19, 2020).

75. Brick, C.; McDowell, M.; Freeman, A.L.J. Risk communication in tables versus text: A registered report randomized trial on “fact boxes." R. Soc. Open Sci. 2020, 7, doi:10.1098/rsos.190876.

76. McDowell, M.; Rebitschek, F.G.; Gigerenzer, G.; Wegwarth, O. A Simple Tool for Communicating the Benefits and Harms of Health Interventions. MDM Policy Pract. 2016, 1, 238146831666536 , doi:10.1177/2381468316665365.

77. Schwartz, L.M.; Woloshin, S. The drug facts box: Improving the communication of prescription drug information. Proc. Natl. Acad. Sci. U. S. A. 2013, 110, 14069-14074, doi:10.1073/pnas.1214646110.

78. Schneider, C.R.; Freeman, A.L.J., Spiegelhalter, D.; van der Linden, S. The effects of quality of evidence communication on perception of public health information about COVID-19: two randomised controlled trials (manuscript in preparation).; 2021;

79. FDA FDA Briefing Document: Moderna COVID-19 Vaccine; 2020;

80. European Medicines Agency COVID-19 Vaccine Moderna; 2020;

81. European Medicines Agency COVID-19 vaccines: development, evaluation, approval and monitoring [Archived] Available online: https://web.archive.org/web/20210101021942/https:/www.ema.europa.eu/en/human-regulatory/overview/publichealth-threats/coronavirus-disease-covid-19/treatments-vaccines/covid-19-vaccines-development-evaluation-approvalmonitoring (accessed on Feb 26, 2021).

82. CDC Understanding and Explaining mRNA COVID-19 Vaccines [Archived] Available online: https://web.archive.org/web/20210106092135/https://www.cdc.gov/vaccines/covid-19/hcp/mrna-vaccine-basics.html (accessed on Feb 26, 2021).

83. British Society for Immunology How vaccines work Available online: https://www.immunology.org/celebratevaccines/public-engagement/guide-childhood-vaccinations/how-vaccines-work (accessed on Feb 26, 2021).

84. O'Connor, A.M. Validation of a Decisional Conflict Scale. Med. Decis. Mak. 1995, 15, 25-30, doi:10.1177/0272989X9501500105.

85. Quintana, D.S.; Williams, D.R. Bayesian alternatives for common null-hypothesis significance tests in psychiatry: A nontechnical guide using JASP. BMC Psychiatry 2018, 18, 178, doi:10.1186/s12888-018-1761-4.

86. Jarosz, A.F.; Wiley, J. What are the odds? A practical guide to computing and reporting bayes factors. J. Probl. Solving 2014, 7, 2-9, doi:10.7771/1932-6246.1167.

87. Public Health England What to expect after your COVID-19 vaccination [Archived] Available online: https://web.archive.org/web/20210224172559/https://www.gov.uk/government/publications/covid-19-vaccination-what-toexpect-after-vaccination/what-to-expect-after-your-covid-19-vaccination\#can-you-give-covid-19-to-anyone-if-you-havehad-the-vaccine (accessed on Feb 26, 2021).

88. Public Health England Even if you've had your \#COVID19 \#vaccine, it's important that you continue to follow national restrictions [tweet] 2021.

89. Marcus, G.E.; Neuman, W.R.; MacKuen, M.B. Measuring Emotional Response: Comparing Alternative Approaches to Measurement. Polit. Sci. Res. Methods 2017, 5, 733-754, doi:10.1017/psrm.2015.65.

90. Wood, T.; Porter, E. The Elusive Backfire Effect: Mass Attitudes' Steadfast Factual Adherence. Polit. Behav. 2019, 41, 135-163, doi:10.1007/s11109-018-9443-y.

91. European Centre for Disease Prevention Catalogue of interventions addressing vaccine hesitancy; Stockholm, 2017; 
92. Penţa, M.A.; Băban, A. Message Framing in Vaccine Communication: A Systematic Review of Published Literature. Health Commun. 2018, 33, 299-314, doi:10.1080/10410236.2016.1266574.

93. O'Neill, J. The COVID-19 vaccine, informed consent and the recruitment of volunteers. J. Med. Ethics blog 2020.

94. Larson, H.J.; Heymann, D.L. Public health response to influenza A(H1N1) as an opportunity to build public trust. JAMA - J. Am. Med. Assoc. 2010, 303, 271-272.

95. Balog-Way, D.H.P.; McComas, K.A. COVID-19: Reflections on trust, tradeoffs, and preparedness. J. Risk Res. 2020, 23, 1-11, doi:10.1080/13669877.2020.1758192.

96. Betsch, C.; Böhm, R.; Korn, L.; Holtmann, C. On the benefits of explaining herd immunity in vaccine advocacy. Nat. Hum. Behav. 2017, 1, 0056, doi:10.1038/s41562-017-0056.

97. Betsch, C.; Böhm, R. Moral values do not affect prosocial vaccination. Nat. Hum. Behav. 2018, 2, 881-882.

98. Troiano, G.; Nardi, A. Vaccine hesitancy in the era of COVID-19. Public Health 2021, doi:10.1016/j.puhe.2021.02.025.

99. Paterson, P.; Meurice, F.; Stanberry, L.R.; Glismann, S.; Rosenthal, S.L.; Larson, H.J. Vaccine hesitancy and healthcare providers. Vaccine 2016, 34, 6700-6706, doi:10.1016/j.vaccine.2016.10.042.

100. Ansell, B.; Bauer, M.W.; Gingrich, J.; Stilgoe, J. Coping With Covid: Two-Wave Survey Available online: https://rpubs.com/benwansell/729135 (accessed on Mar 10, 2021).

101. Nuki, P. Oxford's vaccine is a positive British success story, projecting our soft power around the world. Telegr. 2020. 